\title{
O uso do intensificador de imagem transoperatório na remoção de dente incluso devido lesão cística extensa: Relato de caso clínico
}

The use of the trans operative image intensifier in the removal of an unerupted tooth due to an extensive cystic lesion: Clinical case report

El uso del intensificador de imágenes transoperatorio en la extracción de un diente no erupcionado debido a una extensa lesión quística: Informe de un caso clínico

Recebido: 21/07/2021 | Revisado: 27/07/2021 | Aceito: 30/07/2021 | Publicado: 05/08/2021

Kariana Wan-Dall Gonçalves

ORCID: https://orcid.org/0000-0003-4460-6453 Erasto Gaertner Cancer Center, Brazil E-mail: karianawg@gmail.com

Bruna da Fonseca Wastner ORCID: https://orcid.org/0000-0002-9056-3733 Erasto Gaertner Cancer Center, Brazil Universidade Federal do Paraná, Brazil E-mail: bucobw@gmail.com Vanessa Einsfeld

ORCID: https://orcid.org/0000-0002-8238-6415 Erasto Gaertner Cancer Center, Brazil

E-mail: vaneeinsfelodontologia@gmail.com

William Phillip Pereira da Silva ORCID: https://orcid.org/0000-0003-4172-7217 Erasto Gaertner Cancer Center, Brazil São Paulo State University, Brazil E-mail: william_phillip@hotmail.com

Larissa Gabriele Campos ORCID: https://orcid.org/0000-0001-5816-6300 São Paulo State University, Brazil E-mail: larissa.g.campos@unesp.br Leonardo Faverani ORCID: https://orcid.org/0000-0003-2249-3048 São Paulo State University, Brazil E-mail: leonardo.faverani@unesp.br

Mara Albonei Dudeque Pianovski ORCID: https://orcid.org/0000-0003-0260-8506 Erasto Gaertner Cancer Center, Brazil Universidade Federal do Paraná, Brazil.

E-mail: mpianovski@erastogaertner.com.br

Fernando Luiz Zanferrari

ORCID: https://orcid.org/0000-0001-6286-7126 Erasto Gaertner Cancer Center, Brazil E-mail: flzodonto@gmail.com Laurindo Moacir Sassi

ORCID: https://orcid.org/0000-0002-9333-2498 Erasto Gaertner Cancer Center, Brazil E-mail:sassilaurindo@gmail.com

\section{Resumo}

O diagnóstico tardio de lesões císticas odontogênicas é comum devido o seu crescimento assintomático. Dentre os tratamentos propostos na literatura, atualmente a descompressão ou a marsupialização é indicada para diminuir o tamanho do cisto, para posterior enucleação completa da lesão. A utilização do intensificador de imagem durante o transoperatório, é comumente realizado para orientação e localização de corpos estranhos, projéteis de arma de fogo ou agulhas fraturadas, sendo dificilmente relatado em estudos para auxílio no tratamento de cistos odontogênicos. O presente artigo tem como objetivo relatar um caso clínico de uma paciente jovem, diagnosticada com ceratorcisto odontogênico, a qual foi submetida ao tratamento de marsupialização e posterior enucleação cística. Durante o transoperatório, houve dificuldade localização do dente incluso associado a lesão, devido ao grande deslocamento para a base da mandíbula. Sendo assim utilizado o intensificador de imagem para a localização e remoção do dente. Complicações transoperatórias são comuns de ocorrer, principalmente tratando-se de lesões císticas extensas, devido o grau de dificuldade. A utilização de técnicas e equipamentos que favoreçam benefícios de facilitar e agilizar o 
procedimento cirúrgico acarretam a diminuição de morbidades ao paciente. A utilização do intensificador de imagem, apresenta-se viável em situações incomuns, tanto para localização de corpos estranhos, quanto dentes inclusos em localizações atípicas.

Palavras-chave: Cistos odontogênicos; Fluoroscopia; Maxilares.

\begin{abstract}
The late diagnosis of odontogenic cystic lesions is common due to their asymptomatic growth. Among the treatments proposed in the literature, currently the decompression or marsupialization is indicated to reduce the size of the cyst, for subsequent complete enucleation of the lesion. The use of image intensifier during the transoperative period is commonly performed for orientation and localization of foreign bodies, firearm projectiles or fractured needles, and is hardly reported in studies to aid in the treatment of odontogenic cysts. The present article aims to report a clinical case of a young patient diagnosed with odontogenic keratorcyst, who underwent marsupialization treatment and subsequent cyst enucleation. During the transoperative, it was difficult to locate the unerupted tooth associated with the lesion, due to the large displacement to the base of the mandible. Thus, the image intensifier was used to locate and remove the tooth. Transoperative complications are common, especially when dealing with extensive cystic lesions, due to the degree of difficulty. The use of techniques and equipment that provide benefits in facilitating and speeding up the surgical procedure leads to a reduction in patient morbidity. The use of image intensifier is feasible in unusual situations, both for the location of foreign bodies and teeth in atypical locations.
\end{abstract}

Keywords: Odontogenic cysts; Fluoroscopy; Jaw.

\title{
Resumen
}

El diagnóstico tardío de las lesiones quísticas odontogénicas es frecuente debido a su crecimiento asintomático. Entre los tratamientos propuestos en la literatura, actualmente está indicada la descompresión o marsupialización para reducir el tamaño del quiste, para posteriormente enuclear completamente la lesión. La utilización del intensificador de imágenes durante el período transoperatorio se realiza comúnmente para la orientación y localización de cuerpos extraños, proyectiles de armas de fuego o agujas fracturadas, y apenas se informa en los estudios de ayuda en el tratamiento de quistes odontogénicos. El presente artículo tiene como objetivo reportar un caso clínico de un paciente joven, diagnosticado de queratorquiste odontogénico, que fue sometido a tratamiento de marsupialización y posterior enucleación quística. Durante el transoperatorio, hubo dificultad para localizar el diente no erupcionado asociado a la lesión, debido al gran desplazamiento hacia la base de la mandíbula. Así, se utilizó el intensificador de imágenes para localizar y extraer el diente. Es frecuente que se produzcan complicaciones transoperatorias, especialmente cuando se trata de lesiones quísticas extensas, debido al grado de dificultad. El uso de técnicas y equipos que aportan beneficios para facilitar y acelerar el procedimiento quirúrgico conduce a una disminución de la morbilidad del paciente. El uso del intensificador de imagen es factible en situaciones inusuales, tanto para la localización de cuerpos extraños, como de dientes incluidos en localizaciones atípicas.

Palabras clave: Quistes odontogénicos; Fluoroscopía; Maxilares.

\section{Introdução}

O desenvolvimento das lesões císticas odontogênicas possuem características de crescimento lento e indolor, sendo geralmente diagnosticados através de exames rotineiros ou de uma maneira tardia. Os principais cistos odontogênicos associados a dentes inclusos, como os terceiros molares, são o cisto dentígero e o ceratocisto odontogênico, os quais são caracterizados pela essa evolução silenciosa (Güven et al., 2000; Vigneswaran \& Shilpa, 2015), e quando diagnosticados tardiamente, apresentam extensas lesões, comprometendo grande parte do seio maxilar ou todo o ramo ascendente da mandíbula, incluindo o processo condilar e coronoide (Shear, 2003). A evolução dos cistos odontogênicos pode causar reabsorção ou expansão óssea, fraturas patológicas, injúrias nervosas, deslocamento ou reabsorção (mais raro) radicular dos dentes adjacentes (Deus et al., 2021; Montevecchi et al., 2012; Shear, 2003). A possibilidade de malignização dos cistos reforça mais ainda a importância da sua remoção (Gay-Escoda et al., 2015; Johnson et al., 1994; Panneerselvam et al., 2017; Takahashi et al., 2020).

O dente envolvido pelo cisto, com o passar do tempo e o consequente aumento da lesão, pode ter sua erupção inibida, assim como ser empurrado e adquirir posições atípicas (Mintz et al., 2001; Tümer et al., 2002). Ademais, quanto maior o nível de impacção do dente, maior o risco de complicações (Tsamis et al., 2018). Radiografias panorâmicas convencionais e tomografias computadorizadas auxiliam na localização dos dentes inclusos e no planejamento cirúrgico, porém, no transoperatório, pode haver deslocamento do dente da posição inicial bem como dificuldade na localização exata do dente, 
dificultando o procedimento (Deus et al., 2021; Jihong \& Congfa, 2014). Uma opção para auxílio transoperatório muito utilizado na ortopedia é o uso do intensificador de imagem, um dispositivo que emite radiação contínua de baixa dose por meio de um fluoroscópio (dos Santos Sanches et al., 2021; Mulinari-Santos et al., 2018). Este dispositivo permite obter imagens dinâmicas em tempo real das estruturas internas do paciente, sem que haja distorção das mesmas, o que pode auxiliar na localização de corpos estranhos como agulhas fraturadas e dentes inclusos dentro do complexo maxilofacial (de Queiroz et al.; dos Santos Sanches et al., 2021; Park et al., 2012; Rajkumar et al., 2014). Visando otimizar o tempo cirúrgico e preservar estruturas anatômicas adjacentes, a técnica de localização de corpos estranhos pelo intensificador de imagem demonstra ser uma excelente opção (de Queiroz et al.; dos Santos Sanches et al., 2021). Porém, sua utilização para a localização transcirúrgica de um dente incluso com severo deslocamento associado a cistos não apresenta relatos na literatura. Sendo assim, o objetivo deste artigo é demonstrar o uso do intensificador de imagem na localização e remoção de dente incluso associado a ceratocisto odontogênico extenso.

\section{Metodologia}

O desenvolvimento do estudo retrospectivo, descritivo e observacional, seguiu as diretrizes para a publicação, sem a identificação do paciente e com autorização mediante a assinatura do Termo de Consentimento Livre e Esclarecido (TCLE), seguindo as diretrizes do comitê de ética e pesquisa e Declaração de Helsinque. O estudo de relato de caso clínico no formato de nota técnica, apresenta a utilização do intensificador de imagem durante o ato operatório, para localização de um dente associado a uma grande lesão cística. Os dados epidemiológicos, história da doença e registos fotográficos foram coletados através do prontuário físico e eletrônico do paciente. A fundamentação literária foi realizada através da busca de palavraschave (Odontogenic Cysts; Fluoroscopy; Jaw), nas bases de dados (Pubmed, Science Direct e Google Scholar).

\section{Resultados}

Paciente do sexo feminino, leucoderma, 14 anos de idade, apresentava o diagnóstico de ceratocisto odontogênico em mandíbula à esquerda, associado ao terceiro molar incluso (dente 38) (Figura 1). A paciente havia realizado inicialmente uma biópsia incisional para o diagnóstico definitivo, e devido ao tamanho da lesão, foi optado por uma marsupialização. Após 3 meses da marsupialização, houve pouca redução da lesão cística, apresentando poucas áreas de neoformação óssea, com o deslocamento do dente 38 em basilar da mandíbula (Figura 2).

Foi realizado uma nova tomografia computadorizada da região e reconstrução virtual em 3 Dimensões, para o planejamento cirúrgico. O tratamento cirúrgico de enucleação cística com a remoção do dente incluso associado, foi realizado sob anestesia geral. Durante o procedimento cirúrgico, houve uma dificuldade de localização do dente incluso devido a neoformação óssea após a marsupialização inicial. Para auxiliar a localização e afim de evitar desgastes ósseos desnecessários, foi utilizado o auxílio do intensificador de imagem. Foi utilizado um descolador de molt dentro da cavidade cística para guiar a localização nas imagens obtidas pelo intensificador de imagem (Figura 3). Após a obtenção da imagem e a correta localização, foi realizado osteotomia periférica e odontossecção do dente incluso, para sua remoção (Figura 4). 
Figura 1 - Radiografia panorâmica demonstrando a lesão cística (ceratocisto odontogênico) em corpo e ramo mandibular à esquerda, estendendo de região de molares até processo coronoide e condilar. Nota-se dente 38 incluso, associado a lesão, com deslocamento para base de mandibula, assim como áreas de neoformação óssea após a marsupialização inicial.

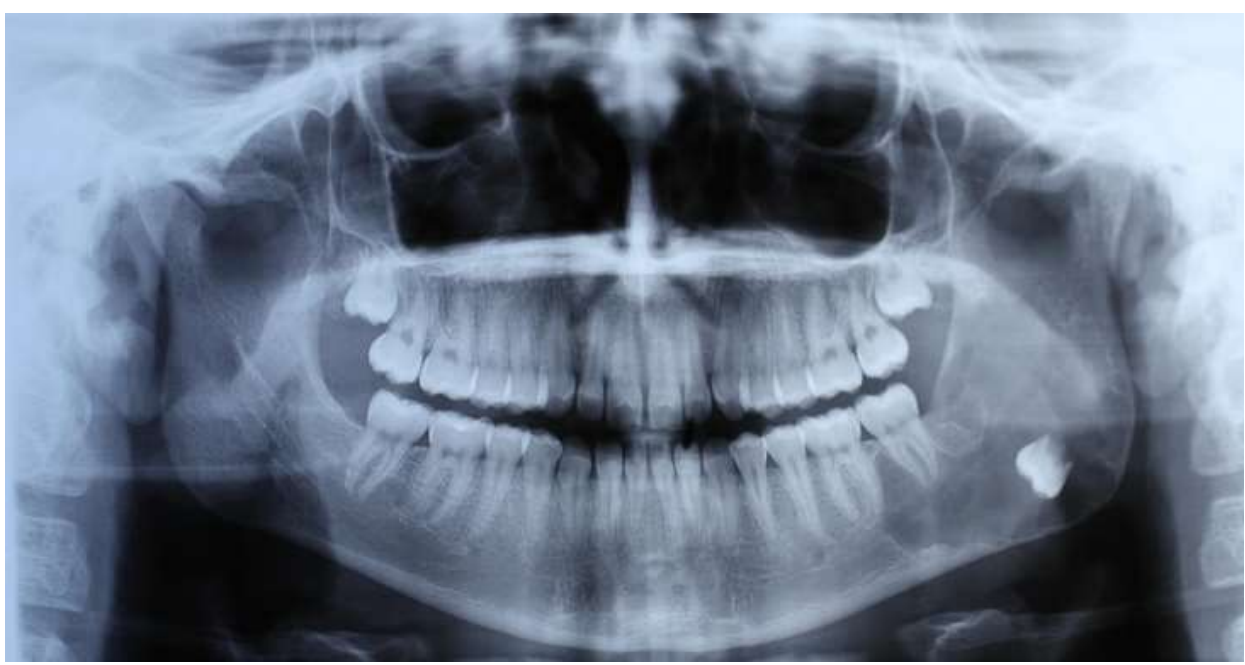

Fonte: Autores.

Figura 2 - A- Imagem de reconstrução em três dimensões, a partir da tomografia computadorizada do paciente, após o tratamento inicial de marsupialização. B - Planejamento virtual, demostrando o deslocamento do dente incluso para a região de base de mandíbula.

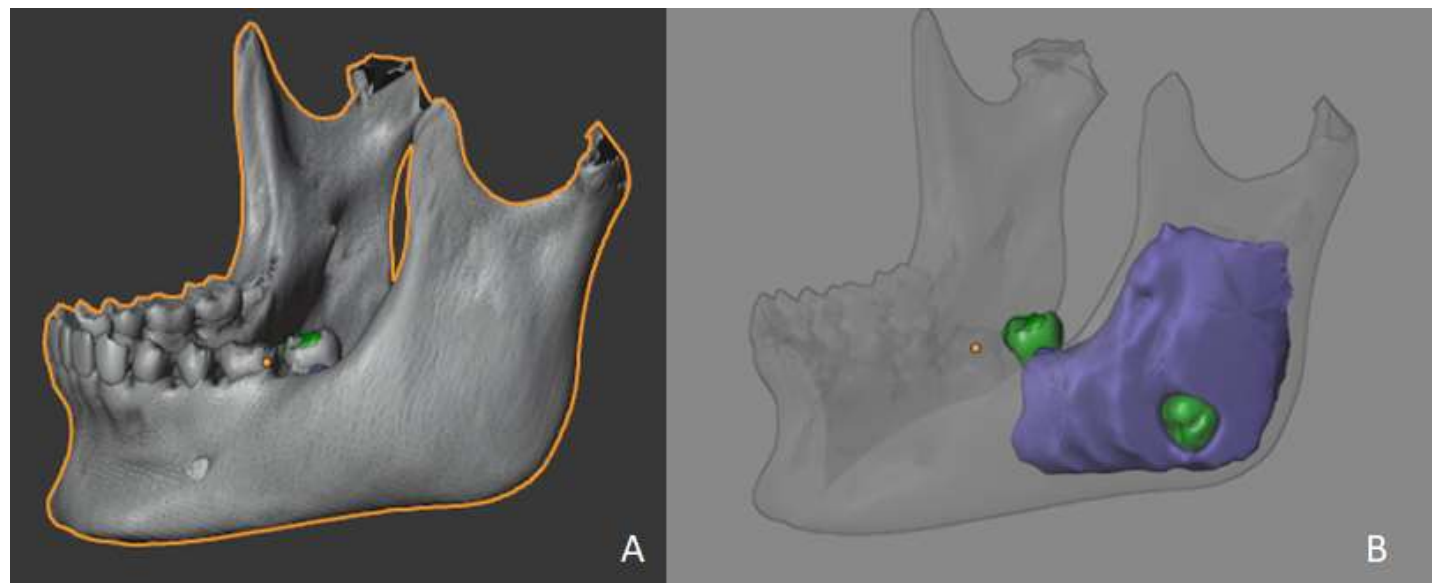

Fonte: Autores. 
Figura 3 - Imagem transoperatória obtida pelo intensificador de imagem, sendo utilizado o descolador de molt como guia para a localização do dente incluso.

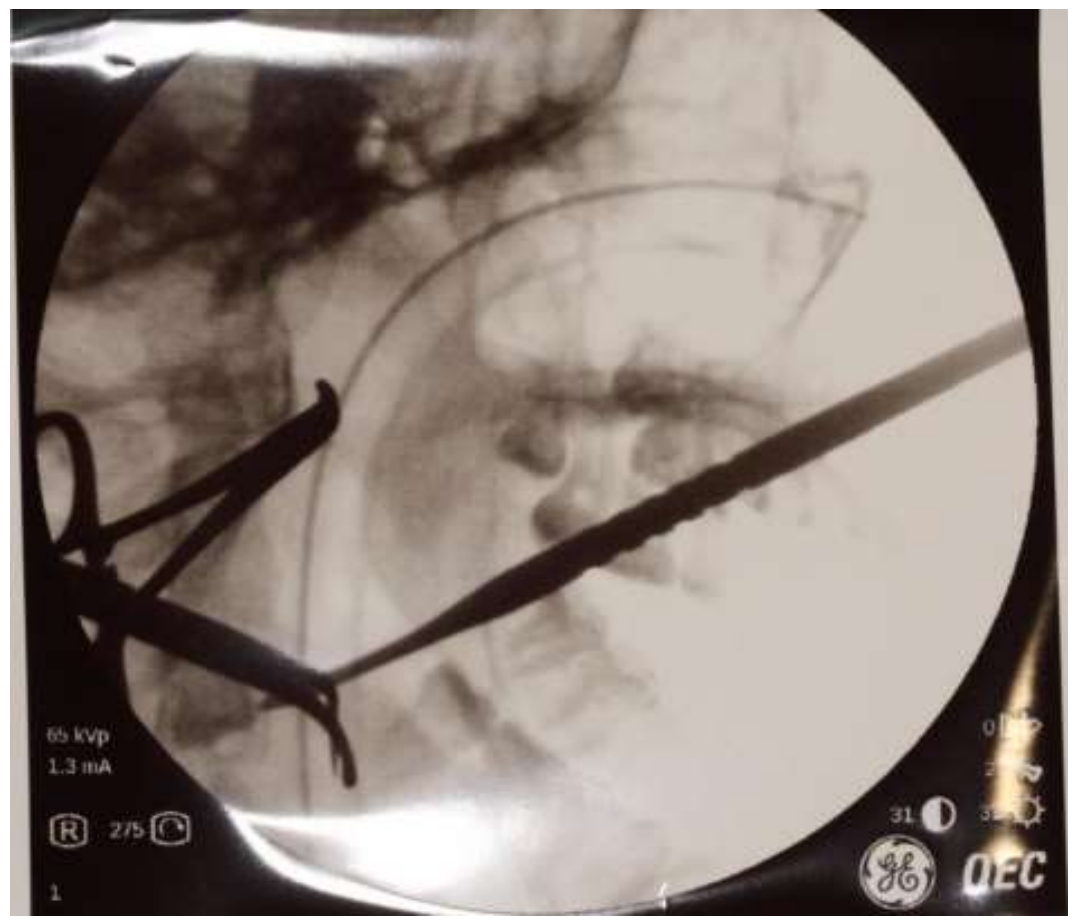

Fonte: Autores.

Figura 4 - (A-D) Sequência ilustrativa da sobreposição da imagem obtida através do uso do intensificador de imagem transoperatório e a reconstrução tridimensional do planejamento virtual prévio.
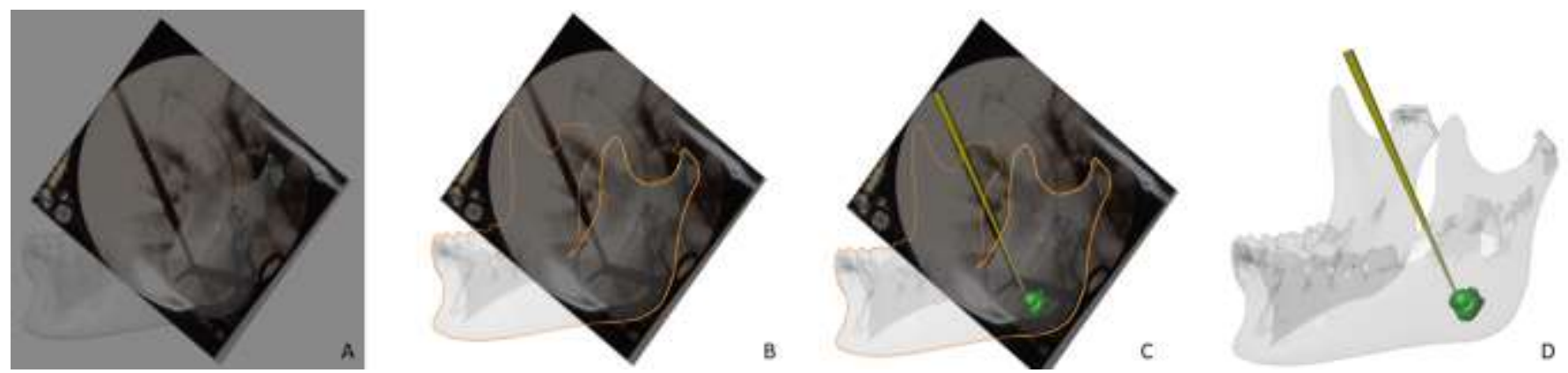

Fonte: Autores.

O acompanhamento pós-operatório imediato, a paciente apresentou-se bem, com edema compatível com o ato cirúrgico e parestesia na região. A paciente segue em acompanhamento pelo serviço, sem sinais de recidiva da lesão, apresentando neoformação óssea na região e com parestesia em região da comissura labial, com sinais clínicas de melhoras após três meses do procedimento cirúrgico.

\section{Discussão}

O tratamento dos cistos odontogênicos extensos, atualmente há um consenso para minimizar danos ao paciente, sendo preconizado tratamento a descompressão ou marsupialização na tentativa da diminuição da lesão e posterior enucleação (Tabrizi et al., 2019). Por muitas vezes, o comportamento após a descompressão ou marsupialização é incerto, devido as características dos cistos, tamanho da lesão e as próprias condições sistêmicas do paciente. A enucleação cística é facilitada 
após esse tratamento, quando há a diminuição da lesão cística (da Silva et al., 2019; Tabrizi et al., 2019). Em nosso estudo, houve uma efetividade no tratamento, ocorrendo uma diminuição do tamanho do cisto e uma neoformação óssea, porém, a região a qual apresentava o dente incluso, apresentou uma neoformação óssea, dificultando a localização e visualização no ato cirúrgico de enucleação cística.

As técnicas de radiologia intervencionista são utilizadas por diversas especialidades médicas com sucesso, especialmente pelos cardiologistas e ortopedistas. Desde a década de 80 já existem relatos do uso do intensificador de imagem em Cirurgia e Traumatologia Bucomaxilofacial em situações como remoção de fragmentos dentários nos espaços faciais, remoção de agulhas fraturadas e de fragmentos de projétil de arma de fogo (Gans et al., 1982). Por fornecer imagens em tempo real (dos Santos Sanches et al., 2021; Faulkner \& Marshall, 1993; Gans et al., 1982; Mulinari-Santos et al., 2018), o intensificador de imagem, apresenta-se como uma opção viável, para a localização de dentes em difícil visualização, como encontrado em nosso estudo.

Uma das vantagens da radiologia intervencionista é a diminuição do tempo cirúrgico, essa técnica foi desenvolvida originalmente por radiologistas, sendo posteriormente adotada por outras especialidades que possuem pouca formação na área (Valentin, 2000). Apesar de ser considerada uma técnica segura em relação aos níveis de radiação, as taxas de dose estão relacionadas ao tempo de exposição e ao número de imagens realizadas. Em alguns casos, é possível causar lesões radioinduzidas tanto nos pacientes quanto em profissionais que utilizam essa ferramenta de forma corriqueira. Um estudo demonstrou que 10 minutos de fluoroscopia com alta dose (200 $\mathrm{mGy} / \mathrm{min})$ é suficiente para causar eritema imediato transiente da pele e que em 15 minutos ocorre depilação temporária. Em exposições mais prolongadas é possível causar descamação, ulceração e até necrose dérmica (CANEVARO, 2009). Portanto, o uso racional é indispensável, assim como as medidas de proteção individuais.

O intensificador de imagem dentro dos sistemas de fluoroscopia fazem a conversão de uma imagem de baixa intensidade em uma imagem minimizada de alta intensidade de brilho (Schueler, 2000). Para trabalhar de forma segura com essa tecnologia, é essencial conhecer sobre seu funcionamento e os riscos e benefícios associados a ela, bem como sobre perfil de radiação espalhada (Canevaro, 2009). Uma característica diferente das imagens produzidas por fluoroscopia é a inversão, visto que corpos radiopacos na radiografia convencional aparecem radiolúcidos, e vice-versa (dos Santos Sanches et al., 2021;

Faulkner \& Marshall, 1993; Gans et al., 1982; Mulinari-Santos et al., 2018). Existe uma curva de aprendizado para compreensão da imagem obtida, por ser uma planificação de um corpo 3D, o que pode ser contornado com o uso de mais de um instrumento-guia, posicionados em angulações diferentes, a fim de complementar a visualização em profundidade (SriPathmanathan, 1990). A utilização de um instrumental como guia para a localização do dente incluso, facilitou a resolução do caso apresentado, possibilitando em poucas tomadas radiográficas a orientação.

\section{Considerações Finais}

Complicações transoperatórias são comuns de ocorrer, principalmente tratando-se de lesões císticas extensas, devido o grau de dificuldade. A utilização de técnicas e equipamentos que favoreçam benefícios de facilitar e agilizar o procedimento cirúrgico acarretam na diminuição de morbidades ao paciente. A utilização do intensificador de imagem, apresenta-se viável em situações incomuns, tanto para localização de corpos estranhos, quanto dentes inclusos em localizações atípicas.

\section{Referências}

Canevaro, L. (2009). Aspectos físicos e técnicos da radiologia intervencionista. Revista Brasileira de Física Médica, 3(1), 101-115. 
Deus, C. B. D. d., Silva, J. V. U., Oliva, A. H. d., Silva, W. P. P. d., Santos, A. M. d. S., Lima Neto, T. J. d., \& Souza, F. Á. (2021). Síndrome de Gorlin Goltz: relato de um caso raro com extenso ceratocisto comprimindo nervo óptico. Research, Society and Development, 10(2), e9410212315. https://doi.org/10.33448/rsd-v10i2.12315

Faulkner, K., \& Marshall, N. W. (1993). The relationship of effective dose to personnel and monitor reading for simulated fluoroscopic irradiation conditions. Health Phys, 64(5), 502-508. https://doi.org/10.1097/00004032-199305000-00007

Gans, B. J., Kallal, R. H., Helgerson, A. C., \& Verona, S. R. (1982). The image intensifier in oral and maxillofacial surgery. Journal of oral and maxillofacial surgery, 40(11), 726-729. https://doi.org/10.1016/0278-2391(82)90146-X

Gay-Escoda, C., Camps-Font, O., López-Ramírez, M., \& Vidal-Bel, A. (2015). Primary intraosseous squamous cell carcinoma arising in dentigerous cyst: Report of 2 cases and review of the literature. Journal of clinical and experimental dentistry, 7(5), e665.

Güven, O., Keskln, A., \& Akal, Ü. K. (2000). The incidence of cysts and tumors around impacted third molars. International journal of oral and maxillofacial surgery, 29(2), 131-135.

Jihong, Z., \& Congfa, H. (2014). The advanced techniques of dentoalveolar surgery. West China Journal of Stomatology, 32(3).

Johnson, L. M., Sapp, J. P., \& McIntire, D. N. (1994). Squamous cell carcinoma arising in a dentigerous cyst. Journal of oral and maxillofacial surgery, 52(9), 987-990.

Mintz, S., Allard, M., \& Nour, R. (2001). Extraoral removal of mandibular odontogenic dentigerous cysts: a report of 2 cases. Journal of oral and maxillofacial surgery, 59(9), 1094-1096.

Montevecchi, M., Checchi, V., \& Bonetti, G. A. (2012). Management of a deeply impacted mandibular third molar and associated large dentigerous cyst to avoid nerve injury and improve periodontal healing: case report. J Can Dent Assoc, 78(2), 59-62.

Mulinari-Santos, G., Bonardi, J. P., Fabris, A. L. d. S., Puttini, I. d. O., Coléte, J. Z., Duailibe-de-Deus, C. B., Faverani, L. P., Garcia Júnior, I. R., \& Souza, F. Á. (2018). Use of an image intensifier for the localization and removal of a foreign body in the lower lip. Archives of Health Investigation, 7(6). https://doi.org/10.21270/archi.v7i6.3008

Panneerselvam, K., Parameswaran, A., Kavitha, B., \& Panneerselvam, E. (2017). Primary intraosseous squamous cell carcinoma in a dentigerous cyst. South Asian journal of cancer, 6(03), 105-117.

Park, S.-S., Yang, H.-J., Lee, U.-L., Kwon, M.-S., Kim, M.-J., Lee, J.-H., \& Hwang, S.-J. (2012). The clinical application of the dental mini C-arm for the removal of broken instruments in soft and hard tissue in the oral and maxillofacial area. Journal of Cranio-Maxillofacial Surgery, 40 (7), 572-578.

Queiroz, S. B. F., Moreira Jr, R., Farina, C. G., Moreira, R., da Silva, A. K. A., \& Coppedê, A. R. USo da fluoroscopia intraoperatória para guiar a colocação de implantes zigomáticos.

Rajkumar, B., Boruah, L. C., Thind, A., Jain, G., \& Gupta, S. (2014). Dental Implant Placement using C-arm CT Real Time Imaging System: A Case Report. The Journal of Indian Prosthodontic Society, 14(1), 308-312.

Silva, Y. S., Stoelinga, P. J., \& da Graça Naclério-Homem, M. (2019). Recurrence of nonsyndromic odontogenic keratocyst after marsupialization and delayed enucleation vs. enucleation alone: a systematic review and meta-analysis. Oral and maxillofacial surgery, 23(1), 1-11.

Santos Sanches, N., Silva, M. C., da Silva, W. P. P., Cervantes, L. C. C., de Lima Neto, T. J., Souza, F. A., Júnior, I. R. G., \& Faverani, L. P. (2021). Auxílio do intensificador de imagem na remoção de agulha gengival curta. Research, Society and Development, 10(1), e41210111889-e41210111889.

Schueler, B. A. (2000). The AAPM/RSNA physics tutorial for residents: general overview of fluoroscopic imaging. Radiographics, 20 (4), 1115-1126. https://doi.org/10.1148/radiographics.20.4.g00j1301115

Shear, M. (2003). Odontogenic keratocysts: clinical features. Oral and maxillofacial Surgery Clinics, 15(3), 335-345.

Sri-Pathmanathan, R. (1990). The mobile X-ray image intensifier unit in maxillofacial surgery. British Journal of Oral and Maxillofacial Surgery, 28(3), 203206. https://doi.org/10.1016/0266-4356(90)90090-8

Tabrizi, R., MsCD, M. R. H. K., \& Jafarian, M. (2019). Decompression or Marsupialization; Which Conservative Treatment is Associated with Low Recurrence Rate in Keratocystic Odontogenic Tumors? A Systematic Review. Journal of Dentistry, $20(3), 145$.

Takahashi, H., Takaku, Y., Kozakai, A., Otsuru, H., Murata, Y., \& Myers, M. W. (2020). Primary intraosseous squamous cell carcinoma arising from a dentigerous cyst of the maxillary wisdom tooth. Case Reports in Oncology, 13(2), 611-616.

Tsamis, C., Rodiou, S., Stratos, A., \& Gkantidis, N. (2018). Removal of a severely impacted mandibular third molar minimizing the risks of compromised periodontium, nerve injury, and mandibular fracture. Quintessence international, 49(1).

Tümer, C., Eset, A. E., \& Atabek, A. (2002). Ectopic impacted mandibular third molar in the subcondylar region associated with a dentigerous cyst: A case report. Quintessence international, 33(3).

Valentin, J. (2000). Avoidance of radiation injuries from medical interventional procedures, ICRP Publication 85. Annals of the ICRP, 30 (2), 7-7.

Vigneswaran, A., \& Shilpa, S. (2015). The incidence of cysts and tumors associated with impacted third molars. Journal of pharmacy \& bioallied sciences, 7(Suppl 1), S251. 